\title{
11 \\ GLOBALISATION AND THE EPIGENETIC LANDSCAPE
}

\author{
PAOLO VINEIS
}

\section{Abstract}

One of the most inspirational books by Tony McMichael has been Human Frontiers, Environments and Disease. In the present chapter, I expand on some of the thoughts that book put forward. There are a few good examples of how environmental changes have modified the genetic make-up of some populations. It is likely, however, that much more important changes have taken place and will take place in humankind's epigenetic landscape, i.e. acquired and transgenerationally transmissible changes in DNA expression. A mounting body of evidence on the importance of epigenetic changes related, for instance, to in utero dietary exposures or deficiencies is now available. The past 30-40 years have seen unprecedented modifications in markets, industrial food availability and other aspects of lifestyle. Is it plausible that these changes could have an enduring effect on humankind's epigenetic landscape? In this chapter, I review some of the existing evidence.

In Human Frontiers, Environments and Disease (McMichael, 2001), McMichael describes in a wonderful way the interplay between human evolution and environmental changes. This chapter is a tribute to that book, with reference to some recent developments in epigenetics that I believe are consistent with Tony's original ideas.

\section{Introduction}

The evolution of living organisms is the expression of different and sometimes opposing forces. The main tension is between the need for stability and the need for change. The first is expressed, for example, by the great structural stability of DNA and of its coding system, conserved almost intact across all species. However, without the ability to change, organisms would not be able 
to adapt to changing environments and their continuous threats. Transposons (transposable elements), crossing over at meiosis and epigenetic changes, are some of the mechanisms that ensure the variety of genetic configurations that allow variation, adaptation and evolution.

It was Barbara McClintock's (awarded the Nobel Prize in 1983: see Figures 11.1 and 11.2) great merit to first understand the relevance of transposable elements and to promote the field of epigenetics after its foundation by Conrad Waddington. The latter defined epigenetics in 1942 as 'the branch of biology which studies the causal interactions between genes and their products, which bring the phenotype into being'. McClintock, on the one hand, hypothesised the existence of 'jumping genes' (transposable elements) through her studies of maize; on the other hand, she first speculated in 1951 that DNA expression was permitted by what today we identify as DNA methylation, or the acetylation of histones:

The progeny of two (such) sister cells are not alike with respect to the types of gene alteration that will occur ... This inactivity or suppression is considered to occur because the genes are 'covered' by other non-genic chromatin materials ... Gene activity may be possible only when a physical change in this covering material allows the reactive components of the gene to be 'exposed' and thus capable of functioning. (McClintock, 1951)

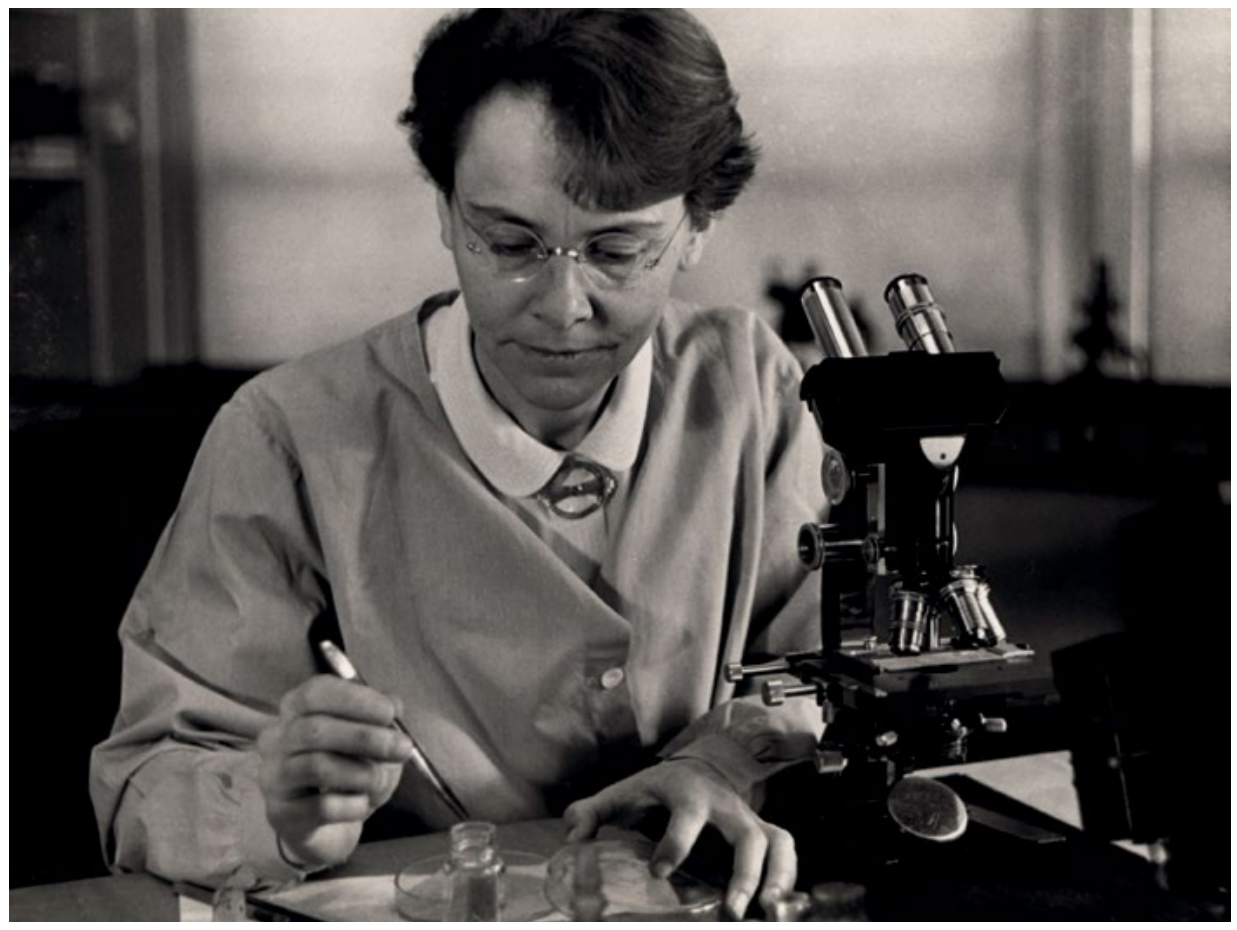

Figure 11.1 Barbara McClintock in the laboratory, 26 March 1947.

Source: profiles.nlm.nih.gov/ps/access/LLBBPP_.jpg, accessed 20 March 2015, courtesy 


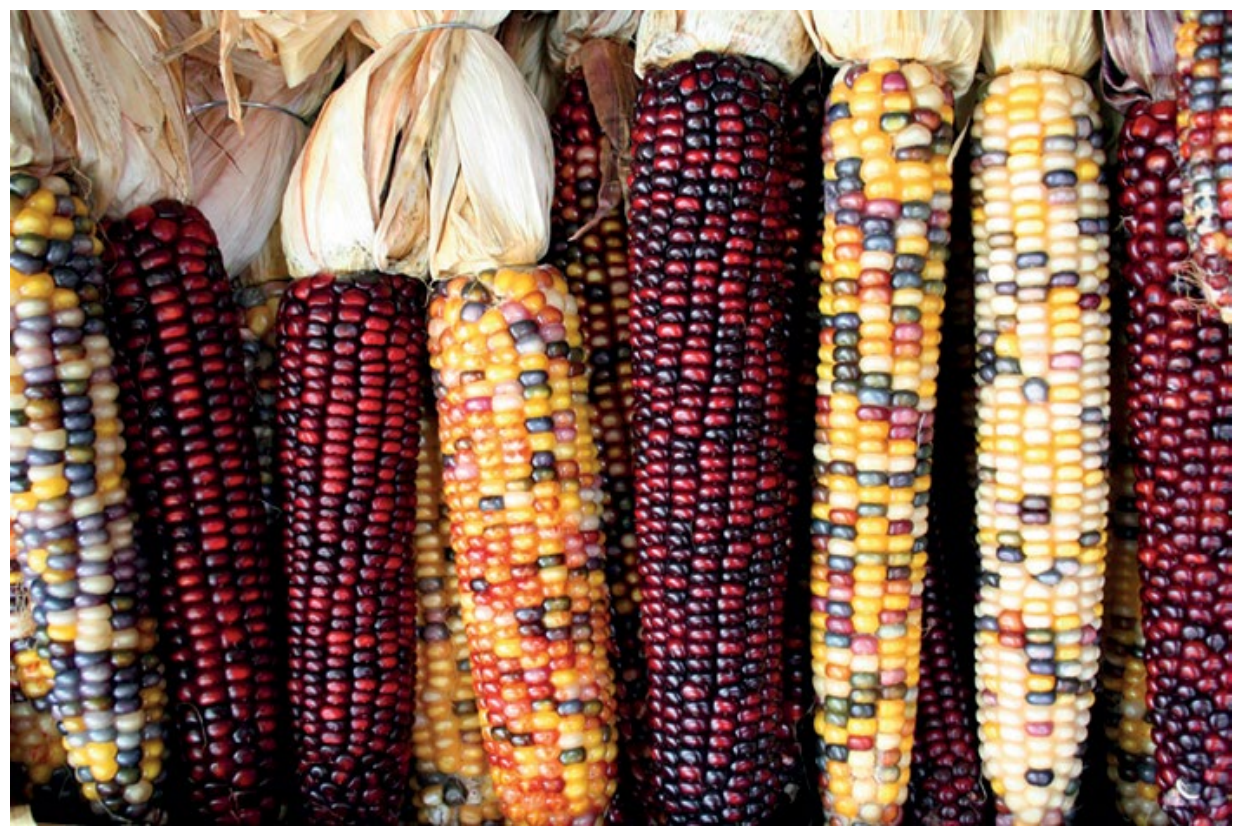

Figure 11.2 Varieties of maize.

Source: Wikimedia commons, en.wikipedia.org/wiki/Blue_corn\#mediaviewer/File:Corncobs.jpg, accessed 14 February 2015.

As a very general rule, environmental stresses tend to increase (epi)genomic instability (via DNA demethylation, for example, or increasing activity of transposable elements), and this is both a mechanism to enhance diversity and respond to threats and a potentially harmful mechanism leading to disease. Over the past two decades, much research has gone into the role of epigenetic changes in the development of diseases like cancer (Feinberg and Tycko, 2004; Salnikow and Zhitkovich, 2008). The most broadly researched and studied epigenetic mechanism is DNA methylation (Christensen and Marsit, 2011). First observed in the early 1980s in studies of carcinogenesis (Feinberg and Tycko, 2004), DNA methylation occurs mainly at CpG dinucleotides, which are found grouped together in promoter regions of around 50 per cent of all human genes (so-called CpG islands). DNA methylation involves the addition of a methyl group to a CpG dinucleotide (Christensen and Marsit, 2011). Methylation of CpG islands can cause gene silencing. If this affects tumour suppressor genes, there may be the induction of cancer. Cancer cells have been seen, in fact, to have unusual patterns of methylation, including both hypermethylation of tumour suppressor genes and hypomethylation of proto-oncogenes or transposable elements (Tabish et al., 2012). 
Another epigenetic mechanism is dysregulation through histone modifications due to the phosphorylation, methylation or acetylation of histone tails (Feinberg and Tycko, 2004), which are predominantly responsible for maintaining nucleosome structures (Luger et al., 1997; Ren et al., 2011). Disruption of normal histone modification mechanisms can cause changes in gene expression and has been observed in the development of some cancers (Luger et al., 1997; Jo et al., 2009). For example, in vitro studies conducted on human cells by Jensen et al. in 2008 described alterations of histone H3 acetylation and changes in gene expression of genes linked to histones, due to the exposure to arsenicals (Jensen et al., 2008). Likewise, in 2009, Zhou et al. (2009) found that arsenite exposure in human lung carcinoma cells led to increased methylation of H3K4 and caused repression of cellular transcription (Luger et al., 1997) (we refer to arsenic because this is one of the environmental exposures best studied from the epigenetic perspective).

A third main mechanism by which epigenetic changes occur is through microRNA (miRNA) expression. miRNAs are described as small, non-coding RNA molecules of approximately 22 nucleotides. miRNAs are involved in genomic and cellular regulation and are thought generally to suppress gene expression. There are many hundreds of miRNAs in humans, and it is believed that up to 30 per cent of genes in mammals are influenced by miRNAs (Luger et al., 1997). Dysregulation of miRNA expression has been suggested to play a role in various diseases (Abdellatif, 2012) such as arsenic-related kidney and bladder cancers (Hoffman and Cairns, 2011).

Exposure to environmental carcinogens and the associated epigenetic changes such as the ones described above can also occur in utero; epigenetic programming may play a major role in embryonic development and the health of an individual in the long term (Christensen and Marsit, 2011). A study conducted on rats by Martínez et al. in 2011 showed that arsenic exposure during gestation resulted in altered patterns of DNA methylation of brain cells affecting memory, thus suggesting that arsenic exposure in utero could have long-standing effects on growth and development (Martínez et al., 2011).

\section{Diabetes, Migrants and Epigenetics}

Birth cohort studies show that maternal undernutrition, low birth weight and rapid post-natal child growth are all associated with an increased risk of diabetes in the offspring. These risks appear to be mediated through epigenetic modification and may be transgenerational. 
The vast majority of low birth weight $(<2500 \mathrm{~g})$ cases occur in the developing world, with the highest incidence in South Asians (31 per cent of live births). The results of The Pune Maternal Nutrition Study, a prospective population-based observational study of rural South Asian women and their offspring, showed that Asian mothers were shorter and thinner than their European counterparts and that full-term South Asian neonates were $\sim 700 \mathrm{~g}$ lighter than the average European. These neonatal differences are accompanied by changes in body composition, with greater reduction in lean muscle tissue than truncal fat.

Birth cohort studies in India confirm the association of maternal undernutrition, low birth weight and thinness in infancy with the subsequent development of impaired glucose tolerance (IGT) and type 2 diabetes (T2D) (Yajnik et al., 2003). Risk of IGT and T2D was highest among South Asians who had shorter mothers or parents with lower body mass index (BMI), or were themselves of low birth weight, thin during infancy but with greater weight gain during childhood and adolescence, independent of their adult BMI. The risk of IGT/T2D was increased six times among South Asians who were in the lowest third of BMI as children but progressed to be in the highest third of BMI as adults, compared with adults who had high BMI as children but became thin adults (Raghupathy et al., 2010). These observations of adverse intrauterine and post-natal exposures in South Asians raise the possibility that epigenetic modification of gene expression may contribute to age-dependent changes in key metabolic genes, and increased susceptibility to T2D.

\section{Diet and Epigenetics}

Diet influences the methylation status of cells in several ways. A major focus of interest has been the 1-carbon metabolism pathway, because this is the main conduit for methyl group donation at a cellular level.

Folate deficiency has been associated with an increased risk of cancer at different sites. The two most commonly hypothesised mechanisms by which folate inadequacy may contribute to carcinogenesis are DNA hypomethylation and uracil misincorporation during DNA synthesis, leading to genomic instability (Crider et al., 2012). While 1-carbon metabolism and folate availability have, to date, been a major focus of attention, it is increasingly apparent that altered DNA methylation is not simply an issue of the bioavailability of methyl groups. For example, the isoflavone, genistein, has profound effects on DNA methylation but has no involvement in 1-carbon metabolism (King-Batoon et al., 2008). 
A striking example of the impact of diet on epigenetic changes is evident in bees. The behaviourally and reproductively distinct queen and worker female castes (same genome) are a result of differential intake of royal jelly (diet-controlled phenotypes) implemented in concert with DNA methylation (brain methylomes). Thus, nutritional control of the reproductive status in honeybees seems to occur via DNA methylation. Australian researchers have recently imitated the effects of royal jelly by turning off the enzyme that labels DNA with methyl groups in bee larvae. These larvae all turned into queens - without any royal jelly (Lyko et al., 2010).

Evidence of the effects of maternal malnutrition on offspring comes from a historical cohort of Dutch individuals whose mothers were exposed during the wartime famine of 1944-45. The offspring of women exposed to malnutrition during early pregnancy were more likely to develop the metabolic syndrome in adulthood compared to the offspring of women pregnant before or after the famine. The effects were dependent on the trimester of gestation in which famine was experienced. Epigenetic analyses of these individuals nearly 60 years later show differential methylation in several genes involved in growth and metabolic control, which are dependent on gender and time of exposure during gestation (de Rooij et al., 2007). Hypomethylation of the promoter of IGF2, a maternally imprinted gene implicated in growth and development, has also been observed in those exposed during the peri-conceptional period relative to unexposed siblings, although the effect is small (Heijmans et al., 2008 ).

In other more recently established cohorts, individuals exposed in utero and during infancy to the Nigerian civil war famine of 1968-70 were at increased risk of hypertension, IGT and being overweight about 40 years later (Barouki et al., 2012). Similarly, women exposed during gestation or early childhood to the 1959-61 famine in China are reported to have a greater risk of metabolic syndrome (Barouki et al., 2012).

\section{Can a 'Healthy' Diet Stabilise the Epigenome?}

We have conducted a randomised dietary intervention study that consisted of enrichment with flavonoids, derived mainly from cruciferous vegetables. We then investigated the methylation status of the promoter regions of cyclin-dependent kinase inhibitor 2A, methylenetetrahydrofolate reductase, Ras-association domain family 1 isoform $\mathrm{A}$, mutL homologue 1 genes and of the LINE-1 repetitive sequence (Scoccianti et al., 2011). LINE-1 transposable elements are a surrogate marker for global DNA methylation levels, and loss of methylation in LINE-1 has been associated with risk of cancer and neurological and cardiovascular diseases (Scoccianti et al., 2011). 
In this study, we observed increased global methylation in peripheral blood cells among the participants, consistent with a role of DNA methylation in controlling retrotransposon mobility by lowering their activities and consequently stabilising the genome. We also observed a decrease of inter-individual methylation levels for three of the panel of five genes after dietary intervention. Together, the increase in global methylation and the decrease of dispersion in the distribution of individual methylation levels may reflect a form of increased epigenetic stability. Epigenetic stability could be the counterpart of genetic stability and may play a role in cancer prevention, since it has been shown that loss of genetic stability promotes tumour progression.

\section{Globalisation and the Epigenetic Landscape}

There are different definitions of globalisation, but a common theme is 'the process of extending social relations across world space'. Such extensions arise from the movements of people, things and ideas (en.wikipedia.org/wiki/ Globalization).

If we consider some of the evidence available on both genetic and (possibly) epigenetic changes in populations, we find that these often come from migrations: lactose tolerance, skin colour and (possibly) diabetes in Asian migrants are examples. One of the features of globalisation is certainly mass migration, but there is much more to it. In fact, it seems that existing populations - as we have known over the last centuries - are the product of long-term adaptation to local circumstances, like the characteristic phenotypes of pygmies, Watusi or Eskimos, probably resulting from a combination of genetic selection and epigenetic features. Local circumstances are now changing very rapidly, and it is difficult to imagine that this will not impact, if not on the genetic make-up, almost certainly on the epigenetic landscape of many population groups.

An example of a rather dramatic secular change is the constantly decreasing age at menarche in girls, which parallels increasing height. These changes are likely to be mediated by epigenetic mechanisms (Demetriou et al., 2013). In addition, as explained by Gluckman and Hanson in their book, Mismatch (Gluckman and Hanson, 2006), the earlier and earlier sexual maturation of girls is mismatched with psychosocial maturation, which is more and more delayed in both girls and boys. The global market exploits both the 'infantilisation' of society and earlier sexual maturation to increase consumerism and target the marketing of commodities for pre-teens. 


\section{Conclusion}

The epigenetic landscape of many population groups is likely to be affected by globalisation. Changes are related to global economic and environmental phenomena, mass migration and the extension to the whole world of features characteristic of Western societies, such as physical inactivity, tobacco smoking, regular alcohol consumption, energy-dense foods and chemical contamination (e.g. endocrine disruptors). The likely consequences of these changes include the spreading epidemic of obesity and diabetes, the spread of cancer to African countries and the decreasing age at menarche in girls.

New insights from epigenetic research might influence policy and practice for example, by demonstrating mechanisms by which environmental factors influence disease, thus providing stronger evidence for policymakers and for civil society. Indeed, showing how epigenetic changes are linked to specific exposures could be more powerful than statistical associations between risk factors and disease, and might lead to innovative legislation. The study of the life-course, transgenerational epigenetic consequences of globalisation should be a top priority for a 21st-century research agenda on environmental diseases.

\section{References}

Abdellatif, M. 2012. Differential expression of microRNAs in different disease states. Circulation Research 110, 638-50.

Barouki, R., Gluckman, P.D., Grandjean, P., Hanson, M. \& Heindel, J.J. 2012. Developmental origins of non-communicable disease: implications for research and public health. Environmental Health 11, 42 doi: 10.1186/1476069X-11-42.

Christensen, B.C. \& Marsit, C.J. 2011. Epigenomics in environmental health. Frontiers in Genetics 2, 28-44.

Crider, K.S., Yang, T.P., Berry, R.J. \& Bailey, L.B. 2012. Folate and DNA methylation: a review of molecular mechanisms and the evidence for folate's role. Advances in Nutrition 3, 21-38.

de Rooij, S.R., Painter, R.C., Holleman, F., Bossuyt, P.M. \& Roseboom, T.J. 2007. The metabolic syndrome in adults prenatally exposed to the Dutch famine. American Journal of Clinical Nutrition 86, 1219-24. 
Demetriou, C.A., Chen, J., Polidoro, S., van Veldhoven, K., Cuenin, C., Campanella, G., et al. 2013. Methylome analysis and epigenetic changes associated with menarcheal age. PLoS One 8, e79391.

Feinberg, A.P. \& Tycko, B. 2004. The history of cancer epigenetics. Nature Reviews Cancer 4, 143-53.

Gluckman, P. \& Hanson, M. 2006. Mismatch - The Lifestyle Diseases Timebomb. Oxford University Press, Oxford, UK.

Heijmans, B.T., Tobi, E.W., Stein, A.D., Putter, H., Blauw, G.J., Susser, E.S., et al. 2008. Persistent epigenetic differences associated with prenatal exposure to famine in humans. Proceedings of the National Academy of Sciences (USA) 105, 17046-9.

Hoffman, A.M. \& Cairns, P. 2011. Epigenetics of kidney cancer and bladder cancer. Epigenomics 3, 19-34.

Jensen T.J., Novak P., Eblin K.E., Gandolfi A.J., Futscher B.W. 2008. Epigenetic remodeling during arsenical-induced malignant transformation. Carcinogenesis. 29(8):1500-8.

Jo, W., Ren, X., Chu, F. Aleshin M., Wintz H., Burlingame A., Smith M.T., Vulpe C.D., Zhang L. 2009. Acetylated H4K16 by MYST1 protects UROtsa cells from arsenic toxicity and is decreased following chronic arsenic exposure. Toxicology and Applied Pharmacology 241, 294-302.

King-Batoon, A., Leszczynska, J.M. \& Klein, C.B. 2008. Modulation of gene methylation by genistein or lycopene in breast cancer cells. Environmental and Molecular Mutagenesis 49, 36-45.

Luger, K., Mader, A.W., Richmond, R.K., Sargent, D.F. \& Richmond, T.J. 1997. Crystal structure of the nucleosome core particle at 2.8 A resolution. Nature $389,251-60$.

Lyko, F., Foret, S., Kucharski, R., Wolf, S., Falckenhayn, C. \& Maleszka, R. 2010. The honey bee epigenomes: differential methylation of brain DNA in queens and workers. PLoS Biology 8, e1000506.

McClintock B. 1951. Chromosome Organization and Genic Expression. Cold Spring Harbor Symposia on Quantitative Biology 16,: 13-47.

McMichael, A.J. 2001. Human Frontiers, Environments and Disease: Past Patterns, Uncertain Futures. Cambridge University Press, Cambridge, UK. 
Martínez, L., Jiménez, V., García-Sepúlved, C., Ceballos, F., Delgado, J.M., NiñoMoreno, P., et al. 2011. Impact of early developmental arsenic exposure on promotor CpG-island methylation of genes involved in neuronal plasticity. Neurochemistry International 58, 574-81.

Raghupathy, P., Raghupathy, P., Antonisamy, B., Geethanjali, F.S., Saperia, J., Leary, S.D., Priya, G., et al. 2010. Glucose tolerance, insulin resistance and insulin secretion in young South Indian adults: relationships to parental size, neonatal size and childhood body mass index. Diabetes Research and Clinical Practice 87, 283-92.

Ren, X., McHale, C.M., Skibola, C.F., Smith, A.H., Smith, M.T. \& Zhang, L. 2011. An emerging role for epigenetic dysregulation in arsenic toxicity and carcinogenesis. Environmental Health Perspectives 119, 11-9.

Salnikow, K. \& Zhitkovich, A. 2008. Genetic and epigenetic mechanisms in metal carcinogenesis and cocarcinogenesis: nickel, arsenic, and chromium. Chemical Research in Toxicology 21, 28-44.

Scoccianti, C., Ricceri, F., Ferrari, P., Cuenin, C., Sacerdote, C., Polidoro, S., et al. 2011. Methylation patterns in sentinel genes in peripheral blood cells of heavy smokers: influence of cruciferous vegetables in an intervention study. Epigenetics 6, 1114-9.

Tabish, A.M., Poels, K., Hoet, P. \& Godderis, L. 2012. Epigenetic factors in cancer risk: effect of chemical carcinogens on global DNA methylation pattern in human TK6 cells. PLoS One 7, e34674.

Zhou, X., Li, Q., Arita, A., Sun, H., Costa, M. 2009. Effects of nickel, chromate, and arsenite on histone 3 lysine methylation. Toxicology and Applied Pharmacology. 236(1):78-84.

Yajnik, C.S., Fall, C.H.D., Coyaji, K.J., Hirve, S.S., Rao, S., Barker, D.J.P., et al. 2003. Neonatal anthropometry: the thin-fat Indian baby. The Pune Maternal Nutrition Study. International Journal of Obesity and Related Metabolic Disorders 27, 173-80. 
This text is taken from Health of People, Places And Planet:

Reflections based on Tony McMichael's four decades of contribution to epidemiological understanding, edited by Colin D. Butler, Jane Dixon and Anthony G. Capon, published 2015 by ANU Press, The Australian National University, Canberra, Australia. 